\title{
The Application of The Double Queue Asymmetric Gated Service Polling Control Theory in Intelligent Traffic System
}

\author{
Yi Fan Zhao ${ }^{1, \mathrm{a}}$,Hong Wei Ding ${ }^{1, \mathrm{a}}$,Meng Yao Wang ${ }^{1, \mathrm{~b}}$, Zhu Guan Liang ${ }^{1, \mathrm{~b}}$, \\ ${ }^{1}$ School of Informatics, Yunnan University, China \\ a,b hw1964@163.com,byakazo@163.com
}

\begin{abstract}
This paper presents a new use of double queues asymmetric gated service polling system in the intelligent traffic light control system.Usually there are more vehicles in main road than minor road,so there are more green light time be needed in the main road.From the computer simulation and theory analysis,we can find that the application of double queues asymmetric gated service polling theory in intelligent traffic system can balance intersections load and set suitable passing time for vehicles to assure the roads open.
\end{abstract}

Keywords:gated service; intelligent traffic; polling; arrive rate; waiting time;

\section{Introduce}

Withing the development of urban economy, the city scale expands unceasingly, the vehicles also become more and more,but when people enjoying the convenient that vehicles bring,at the same time, also meet more and more problems,such as air pollution,noise pollution and traffic congestion. The traffic light in intersections is a very important role to maintain the traffic order, guarantee the road clear. The development of urban lead to more and more vehicles. Ordinary traffic system can not meet the requirement today. So in order to Alleviate the increasing traffic pressure, the intelligent traffic system ${ }^{[1]}$ come.

Intelligent traffic system including the detection of road traffic, according to the intersection traffic flow characteristics and queuing delay, effectively, design a more advanced traffic signal timing scheme in order to improve the efficiency of intersection and so on. The appropriate traffic lights control strategy which can alleviate the traffic congestion is the most important topic in intelligent traffic system.

In this paper we apply the theory of double queues asymmetric gated service polling ${ }^{[2]}$ to intelligent traffic system. Designing a traffic lights system which can adjust the timekeeping time of the traffic light according to the change of the traffic volume. To alleviate traffic pressure, and can save transportation resources.Through reasonable design, the system can realize control length of red,green,yellow light time reasonably and automatically with different arrival rate.

\section{Specific design}


we design a traffic light system which the light time can be adjusted intellectually according to the change of the vehicles arrive rate ${ }^{[3]}$.

Specific design is installing ground loop ${ }^{[4]}$ in all directions 80 meters away from the intersections to count the number of vehicles, and then enter data into the computer central control system to analyze the data and calculate the waiting time through the polling model with the current arrive rate.This result of the calculation been seem as the green light time in this direction.

In this intelligent system,because the application of double queues asymmetric gated service polling theory, the load of main road which has higher arrive rate and minor road which has lower arrive rate balancing well.Considering that there are more vehicles arrive in the main road,so there need longer pass time in main road.

\section{Polling system}

Polling system model ${ }^{[5]}$ consists of a server and $\mathrm{N}$ queues ${ }^{[6]}$. The queues arrival process and the service time are random process in queuing mode.

The double queues asymmetric gated service polling control system made up of a server,a high arrive rate queuel and a low arrive rate queue2. The queue1 and queue 2 adopt the rule which is gated service. Whatever the queue1 and queue2,the server services the current gated information packet with gated service,only, and turn to service anther queue after a conversion time. The information packet arrive during the service time of the queue would be serviced in the nextpollingcycle.This is the principle of double queues asymmetric gated service polling control system. The asymmetry beem bodiedin different arrive rate of the queue1 and queue2

\section{The double queue asymmetric gated service polling model of intelligent traffic lights control system}

This intelligent traffic lights control system is seem as a double queues asymmetric gated service polling control system.For practical purposes,suppose,the main road be seem as a queue with the higher arrival rate of vehicles which is $\lambda_{h}$, the minor be seem as a queue with the lower arrival rate of vehicles which is $\lambda_{o}$.The main road and minor road both adopt the rule which is gate service polling strategy.The $\beta$ means the service rate of the traffic light system.In this intelligent system $\beta$ is 1 , mean there is one vehicle spend $1 \mathrm{~s}$ to pass the waiting line.The conversion rate of traffic light system is the length of yellow light time in this system,and there $\gamma=10$ means that there are $10 \mathrm{~s}$ yellow light.

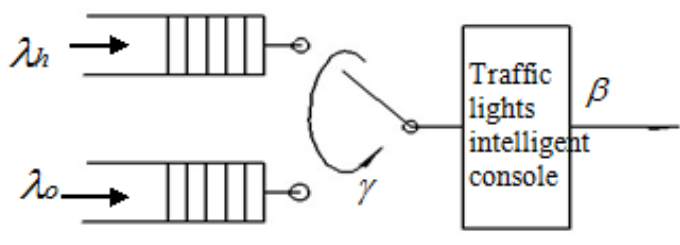

$\beta$ :The servicerate of the trafficelight system $\gamma$ :The conversion rate of the trafficelight system $\lambda_{h}$ : The arrival rate of vehicles in the main road $\lambda_{0}$ : The arrival rate of vehicles in the minor road

Fig 1. The double queues asymmetric gated service polling model of intelligent traffic lights control system

\section{Queuing model and operation mode}

There are 2 queues serviced by one traffic lights console in this intelligent traffic lights control system.Due to the queuing system is a solving process under the discrete time state,so the time axis is divided in time slot $\tau$ (To make analysis into concise,we take $\tau$ as the unit of time)

\subsection{Assumptions}

Each queues has the vehicles arrived into their queue space with independent and identically probability distribution at any time slot the distribution of probability generating function,the mean and variance are $A_{i}(Z) 、 \lambda=A_{i}{ }^{\prime}(1)$, and $\delta_{\lambda}^{2}=A_{i}^{\prime \prime}(1)+\lambda-\lambda^{2}$ respectively. 
The random variable of query conversion time between the two queues is subject to a probability distribution which is mutually independent and identically distributed, the distribution of probability generating function, the mean and variance are $R(Z), \gamma=R^{\prime}(1)$ and $\delta_{\gamma}^{2}=R^{\prime \prime}(1)+\gamma-\gamma^{2}$ respectively.

The random variable of the time that each vehicles pass the crossroads is subject to a probability distribution which is mutually independent and identically distributed, the distribution of probability generating function, the mean and variance are $B(Z) 、 \beta=B^{\prime}(1)$ and $\delta_{\beta}^{2}=B^{\prime \prime}(1)+\beta-\beta^{2}$ respectively.

The queue space is big enough. The number of vehicles would not more than the queue space.

For the vehicles that in the each queue, according to the principle of first-in-first-out.

\subsection{Mean queue length}

Define the variable $g_{1}(1)$ as the number of vehicles in the minor road and main road waiting for passing the interaction while there is the green light in the minor road and the variable $g_{2}(2)$ as the number of vehicles in the minor road and main road waiting for passing the interaction while there is the green light in the main road.then,

$$
\begin{aligned}
& g_{1}(1)=\frac{2 r \lambda_{1}}{1-\rho_{1}-\rho_{2}} \\
& g_{2}(2)=\frac{2 r \lambda_{2}}{1-\rho_{1}-\rho_{2}}
\end{aligned}
$$

\subsection{Mean waiting time}

Then, define the variable $E\left(W_{i}\right)$ as mean waiting time of the vehicles in the minor road and main $\operatorname{road}(\mathrm{i}=1,2)$.According to queue theory, the mean waiting time $E\left(W_{i}\right)$ are expresses as follows:

$$
\begin{aligned}
& E\left(W_{1}\right)=\frac{\left(1+\rho_{1}\right) g_{1}(1,1)}{2 \lambda_{1} g_{1}(1)}-\frac{A_{1}^{\prime \prime}(1)}{2 \lambda_{1}^{2}} \\
& E\left(W_{2}\right)=\frac{\left(1+\rho_{2}\right) g_{2}(2,2)}{2 \lambda_{1} g_{2}(2)}-\frac{A_{2}^{\prime \prime}(1)}{2 \lambda_{2}^{2}}
\end{aligned}
$$

\section{The simulation}

The simulation calculation and theoretical calculation take the same parameters.

Because the main objective in this paper is find a traffic lights control strategy to balance load and alleviate traffic pressure, so the simulations is simulating peak-time road condition that the main road load $\rho_{1}$ is the double of load $\rho_{2}$ in the minor road.

In this system, there has a rang which is stable region. The stable region means in this rang of load, this system is working properly and steadily, would not occur traffic congestion and end up with the traffic system collapse. And there has a rule of the stable region is $\rho_{1}+\rho_{2}<1$.So, in the simulation of peak-time $\left(2 \rho_{1}=\rho_{2}\right)$ as the Fig.2,Fig.3 and Fig.4,the stable region is less than

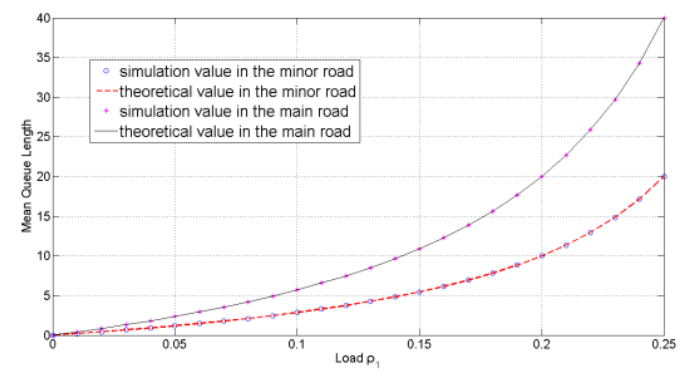

0.25 .

Fig.2 The mean queue length with the system

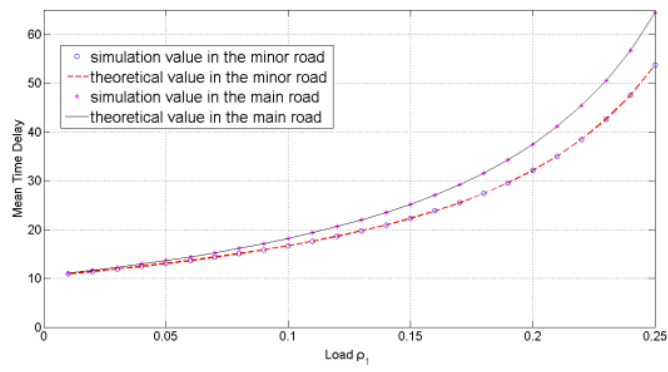

Fig.3 The mean waiting time with the system 
The Fig2 and Fig3 reflect the theoretical value and the simulation values are the same.In those two diagrams we can find that the mean queue length and the mean time delay are proportional to load. Compare main road condition and minor road condition we can find that the mean queue length and the mean time delay of main road is longer than the mean queue length and the mean time delay of minor road under the assumed condition that $2 \rho_{1}=\rho_{2}$. So, considering the mean queue length and the mean time delay the longer green light time be need in the main road.

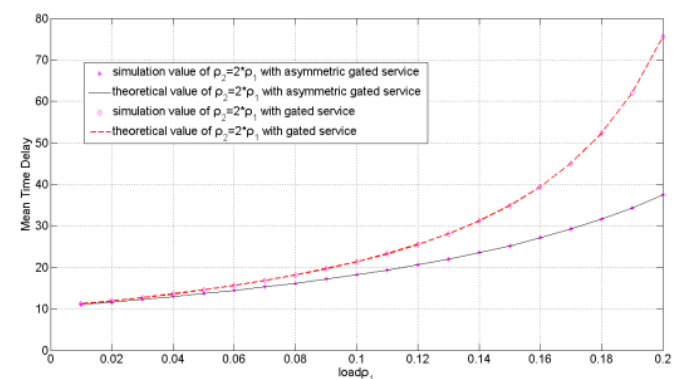

Fig.4 Compare with normal gated service polling

The Fig4 contrasts the mean waiting time under double queues asymmetric gated service polling theory to mean waiting time under normal gated service polling theory. In the main road which $2 \rho_{1}=\rho_{2}$ the double queues asymmetric gated service polling theory manifests the superiority. The double queues asymmetric gated service polling theory has the shorter waiting time and greater reliability than normal gated service polling theory with the same load (simulate $2 \rho_{1}=\rho_{2}$ ).In a real world application, the intelligent traffic system which adopt the double queues asymmetric gated service polling theory can ensure the smooth road better.

\section{Conclusions}

Different road has different arrival rate. Whatever in the main road which has higher load or in the minor road which is low load, the mean queue length and mean time delay increase over the system load.Compared to main road and minor road,because the main road has the higher arrive rate so there has longer waiting time and the longer green light time would be need in the real world application. And by contrasting the simulation of double queues asymmetric gated service polling theory to normal gated service polling theory, the former is presenting advantages in the heavy load. Compute simulating result are consistent with theoretical analyses. Through above analysis, the intelligent traffic light control system which using double queues asymmetric gated service polling theory can do improve the road capacity.

\section{References}

[1]Dimitrakopoulos, G. (2011) Intelligent transportation systems based on internet-connected vehicles: Fundamental research areas and challenges. 11th International Conference on ITS Telecommunications (ITST), Saint Peterburg, 23-25 August 2011, 145-151.

[2]Ding Hongwei, Zhao Dongfeng, Zhao Yifan. Packet delay analysis of continuous-time polling system with M-gated services[A]. Chengdu, P.R.China, August, 2010, Page(s):408-411.

[3] Jan-Pieter Dorsman, S. Borst, O. Boxma, Maria Vlasiou,Markovian polling systems with an application to wireless random-access networks[J].IEEE Trans. Eurandom Prepront Series, 2014, 5(6):1389-2355.

[4]Dimitrakopoulos, G. (2011) Intelligent transportation systems based on internet-connected vehicles: Fundamental research areas and challenges. 11th International Conference on ITS Telecommunications (ITST), Saint Peterburg, 23-25 August 2011, 145-151.

[5] Giambene, G.,Hadzic-Puzovic, S. Nonsaturated Performance Analysis for WiMAX Broadcast Polling Access[J]. IEEE Transactions on Vehicular Technology, 2013, 62(1):306-325.

[6] Qianlin Liu, Dongfeng Zhao, Dongming Zhou. An analytic model for enhancing IEEE 802.11 coordination function media access control protocol[J]. European Transactions on Telecommunications, 2011, 22(6):332-338. 